\title{
The Anion-Selective Pore of the Bestrophins, a Family of Chloride Channels Associated with Retinal Degeneration
}

\author{
Zhiqiang Qu, Li-Ting Chien, Yuanyuan Cui, and H. Criss Hartzell \\ Department of Cell Biology and Center for Neurodegenerative Disease, Emory University School of Medicine, Atlanta, Georgia 30322
}

\begin{abstract}
Mutations in human bestrophin-1 (VMD2) are genetically linked to a juvenile form of macular degeneration and autosomal dominant vitreoretinochoroidopathy. Recently, it has been proposed that bestrophins are $\mathrm{Cl}^{-}$channels and that the putative second transmembrane domain participates in forming the bestrophin pore. However, the structural determinants of $\mathrm{Cl}^{-}$ion permeation through the channel pore are not known. Here we systematically replaced every amino acid in mouse bestrophin-2 (mBest2) between positions 69 and 104 with cysteine. We then measured the effects on the relative permeability and conductance of the channel to $\mathrm{Cl}^{-}$and SCN ${ }^{-}$(thiocyanate) and determined the accessibility of the cysteine-substituted amino acids to extracellularly applied, membrane-impermeant sulfhydryl reagents. Unlike $\mathrm{K}^{+}$channels, the amino acids forming the $\mathrm{mBest} 2$ selectivity filter are not discretely localized but are distributed over $\sim 20$ amino acids within the transmembrane domain. Cysteine-substituted amino acids in the selectivity filter are easily accessible to extracellularly applied sulfhydryl reagents and select for anionic sulfhydryl reagents over cationic ones. Understanding the structure of the anion conduction pathway of bestrophins provides insights into how mutations produce channel dysfunction and may provide important information for development of therapeutic strategies for treating macular degeneration.
\end{abstract}

Key words: ion channel; anion channel; chloride channel; macular degeneration; bestrophin; patch clamp

\section{Introduction}

Age-related macular degeneration is the most common cause of blindness in the United States (Penfold et al., 2001). Although genetic and environmental factors have been identified as factors contributing to its pathogenesis, the basic mechanisms remain poorly understood. To understand the mechanisms better, it is hoped that inherited forms of macular degeneration will provide clues. Best vitelliform macular dystrophy (Best disease) is inherited in an autosomal dominant manner and is linked to mutations in the bestrophin-1 gene (VMD2) (Hartzell et al., 2005; Strauss, 2005). In Best disease, lipofuscin accumulates between the retinal pigment epithelium (RPE) and photoreceptors and within RPE cells and macrophages. The lipofuscin accumulation is thought to be causally related to the subsequent photoreceptor degeneration that ensues (Hartzell et al., 2005). The gene responsible for Best disease was positionally cloned in 1998 from families exhibiting macular degeneration with a juvenile age of onset (Marquardt et al., 1998; Petrukhin et al., 1998). More than 85 human bestrophin-1 (hBest1) mutations are now associated with macular degeneration (White et al., 2000; Weber and Krämer, 2002). In addition, inherited abnormal splice variants of VMD2 are associated with autosomal dominant vitreoretinochoroidopathy (Yardley et al., 2004).

Received Dec. 22, 2005; revised March 9, 2006; accepted April 7, 2006.

This work was supported by National Institutes of Health Grants GM60448 and EY014852, the American Health Assistance Foundation (H.C.H.), and the American Heart Association (Z.Q.)

Correspondence should be addressed to H. Criss Hartzell, Department of Cell Biology, Emory University School of Medicine, 615 Michael Street, 535 Whitehead Building, Atlanta, GA 30322-3030. E-mail: criss.hartzell@emory.edu. DOI:10.1523/JNEUROSCI.5500-05.2006

Copyright $\odot 2006$ Society for Neuroscience $\quad$ 0270-6474/06/265411-09\$15.00/0
It has been speculated that Best disease is linked to a transport defect in the RPE because the hallmark diagnostic feature of Best disease is a decreased slow light peak in the electrooculogram (Wajima et al., 1993). The slow light peak is thought to reflect a Cl conductance in the basolateral membrane of the retinal pigment epithelium (Gallemore et al., 1997). Because Best1 is localized at the basolateral membrane of the RPE (Marmorstein et al., 2000; Bakall et al., 2003), the idea that Bestl is responsible for the basolateral $\mathrm{Cl}$ conductance is particularly attractive.

Bestrophins from several species function as $\mathrm{Ca}$-activated $\mathrm{Cl}$ channels when expressed heterologously (Sun et al., 2002; Qu and Hartzell, 2003, 2004; Tsunenari et al., 2003; Fischmeister and Hartzell, 2004; Qu et al., 2004). Unfortunately, identification of $\mathrm{Cl}$ channels by heterologous expression has a troubled history (Clapham, 1998; Jentsch et al., 2002; Hartzell et al., 2005). There are a number of proteins whose $\mathrm{Cl}$ channel function is doubted, although they induce $\mathrm{Cl}$ currents when overexpressed. Because many $\mathrm{Cl}$ currents are biophysically similar and very few specific blockers have been identified, it is often difficult to be certain that an expressed protein is not simply upregulating an endogenous $\mathrm{Cl}$ channel.

We have shown previously that the anion permeability of mouse Best2 (mBest2) could be altered by mutations of some amino acids in the putative second transmembrane domain (TMD2) (Qu and Hartzell, 2004; Qu et al., 2004). These studies provided the first strong evidence that bestrophins are indeed $\mathrm{Cl}^{-}$channels (Pusch, 2004). Having identified TMD2 as an important region for channel function, we replaced every amino acid between C69 and D104 with cysteine and examined the permeability properties and sensitivity to sulfhydryl reagents to understand the role of TMD2 in anion permeation of mBest2. 


\section{Materials and Methods}

Site-directed mutagenesis and heterologous expression of mBest2. Sitespecific mutations of mBest 2 were made using PCR-based site-directed mutagenesis (Quickchange; Stratagene, La Jolla, CA) as described previously (Qu et al., 2004). mBest2 cDNA [American Type Culture Collection (Manassas, VA); IMAGE clone 4989959; GenBank accession numbers BC031186 and NM_145388] and its mutants in pCMV-SPORT6 vector were transfected into HEK293 cells (American Type Culture Collection) using FuGene-6 transfection reagent (Roche, Indianapolis, IN). Phosphorylated enhanced green fluorescent protein (EGFP) (Invitrogen, Carlsbad, CA) was cotransfected to identify transfected cells. To obtain modest amplitude currents (1-3 nA per cell), 0.05-0.1 $\mu \mathrm{g}$ of mBest2 wild-type or $0.025-0.3 \mu \mathrm{g}$ mBest 2 mutant was used to transfect one 35 $\mathrm{mm}$ culture dish. One day after transfection, cells were trypsinized and replated on glass coverslips for electrophysiological recording. Transfected cells were identified by EGFP fluorescence and used for patchclamp experiments within $3 \mathrm{~d}$ after transfection.

Electrophysiology. Recordings were performed using the whole-cell recording configuration of the patch-clamp technique (Neher, 1992). Patch pipettes were made of borosilicate glass (Sutter Instruments, Novato, CA), pulled by a Sutter Instruments P-2000 puller, and fire polished. Patch pipettes had resistances of 2-3.5 $\mathrm{M} \Omega$ filled with the standard intracellular solution (see below). The bath was grounded via a $3 \mathrm{M} \mathrm{KCl}$ agar bridge connected to a $\mathrm{Ag} / \mathrm{AgCl}$ reference electrode. Solution changes were performed by perfusing the $1 \mathrm{ml}$ chamber at a speed of $\sim 4 \mathrm{ml} / \mathrm{min}$. Experiments were performed with 100 - or 200-ms-duration voltage ramps from -100 to $+100 \mathrm{mV}$. The start-to-start interval was $10 \mathrm{~s}$. Data were acquired by an Axopatch 200A amplifier controlled by Clampex 8.1 via a Digidata 1322A data acquisition system (Molecular Devices, Palo Alto, CA). Experiments were conducted at room temperature $\left(20-24^{\circ} \mathrm{C}\right)$. Liquid junction potentials were measured using the liquid junction potential calculator in Clampex 8.1 to correct $E_{\text {rev }}$ of various ionic conditions. The standard pipette solution contained the following (in $\mathrm{mM}$ ): $146 \mathrm{CsCl}, 2 \mathrm{MgCl}_{2}, 5$ ( $\left.\mathrm{Ca}^{2+}\right)$-EGTA, 8 HEPES, and 10 sucrose, pH 7.3, adjusted with $N$-methyl-D-glucamine. The free $\left[\mathrm{Ca}^{2+}\right]$ in the solution was determined as described by Kuruma and Hartzell (2000). The calculated $\mathrm{Ca}^{2+}$ concentration in the high- $\mathrm{Ca}^{2+}$ intracellular solution was confirmed as $4.5 \mu \mathrm{M}$ by fura- 2 (Invitrogen) measurements using an LS-50B luminescence spectrophotometer (PerkinElmer, Wellesley, MA). The standard extracellular solution contained the following (in mM): $140 \mathrm{NaCl}, 5 \mathrm{KCl}, 2 \mathrm{CaCl}_{2}, 1 \mathrm{MgCl}_{2}, 15$ glucose, and $10 \mathrm{HEPES}, \mathrm{pH}$ 7.3 with $\mathrm{NaOH}$. This combination of solutions set $E_{\text {rev }}$ for $\mathrm{Cl}^{-}$currents to zero, whereas cation currents carried by $\mathrm{Na}^{+}$or $\mathrm{Cs}^{+}$have very positive or negative $E_{\text {rev }}$, respectively. When $\mathrm{Cl}^{-}$was substituted with another anion, $\mathrm{NaCl}$ was replaced on an equimolar basis with $\mathrm{NaX}$, in which $X^{-}$is the substitute anion. Solution osmolarity was $303 \mathrm{mOsm}$ for both intracellular and extracellular solutions. Small differences in osmolarity were adjusted by addition of sucrose.

Sulfhydryl modification. 2-Trimethylammonioethylmethanethiosulfonate bromide (MTSET), sodium [2-sulfonatoethyl] methanethiosulfonate (MTSES) (Toronto Research Chemicals, Toronto, Ontario, Canada), and $\mathrm{N}$-ethylmaleimide (NEM) (Pierce, Rockford, IL) were prepared in water, stored on ice, and used within $90 \mathrm{~min}$. The reagents were diluted to the indicated working concentration immediately before use. Typically, the MTS reagent was applied at a holding potential of $0 \mathrm{mV}$. Every $10 \mathrm{~s}$, the cell was subjected to a voltage pulse consisting of a $200 \mathrm{~ms}$ step to $-100 \mathrm{mV}$, followed by a $200 \mathrm{~ms}$ ramp to $+100 \mathrm{mV}$. The concentration of MTS reagent was selected so that the effect reached a steady-state within 1-5 min. However, with some mutations, such as K289C, that responded very slowly to MTS reagents, it took up to $10 \mathrm{~min}$ for steady state to be achieved. In all cases, the effects of sulfhydryl-modifying reagents were allowed to reach a steady state. When a specific cysteine mutation did not respond or responded weakly to MTS, the S79C mutation was used as a positive control to verify MTS potency.

Analysis of data. Data are expressed as mean \pm SEM. Relative permeability of the channels was determined by measuring the shift in $E_{\mathrm{rev}}$ after changing the bath solution from one containing $151 \mathrm{mM} \mathrm{Cl}^{-}$to another with $140 \mathrm{~mm} \mathrm{X}^{-}$and $11 \mathrm{~mm} \mathrm{Cl}^{-}$, in which $X^{-}$is the substitute anion $(\mathrm{Qu}$ and Hartzell, 2000). The permeability ratio was estimated using the Goldman-Hodgkin-Katz equation: $P_{x} / P_{\mathrm{Cl}}=\left[\mathrm{Cl}^{-}\right]_{\mathrm{i}} /\left(\left[X^{-}\right]_{\mathrm{o}}\right.$ $\left.\exp \left(\Delta E_{\text {rev }} F / R T\right)\right)-\left[\mathrm{Cl}^{-}\right]_{\mathrm{o}} /[X]_{\mathrm{o}}$, where $\Delta E_{\text {rev }}$ is the difference between the reversal potential with the test anion $X^{-}$and that observed with symmetrical $\mathrm{Cl}^{-}$, and $F, R$, and $T$ have their normal thermodynamic meanings. OriginPro 7.0 (Microcal Software, Northampton, MA) was used for calculations and graphical presentation.

\section{Results \\ Identification of two critical amino acid clusters by cysteine mutagenesis}

Tsunenari et al. (2003) proposed that hBest1 has four transmembrane domains based on hydropathy and insertion of $\mathrm{N}$-glycosylation sites and tobacco etch virus (TEV) protease cleavage sites (Fig. 1A). The putative second transmembrane domain is likely to be involved in formation of the $\mathrm{Cl}^{-}$-selective pore because it is very highly conserved among different bestrophins and because mutations of several residues in this region alter the relative anion permeability and conductance of the channel (Qu and Hartzell, 2004; Qu et al., 2004). To derive a model of the pore region of bestrophins, we measured the effects of cysteine mutagenesis of all amino acids extending from C69 to D104. For simplicity, we will refer to this region simply as C69D104. This region includes the putative membrane domain that is consistently predicted by a variety of algorithms to be centered around Y85 plus flanking amino acids that are expected to be located in either cytoplasmic or extracellular loops. We also made mutations in most of the arginine and lysine residues within or near other predicted transmembrane domains with the rationale that positively charged amino acids might be involved in stabilizing anions in the pore.

Within C69-D104, there were six residues that, when changed to cysteine, produced no current. Four of these residues (S74, L75, I76, and P77, "the SLIP cluster") were clustered together. Replacement of I76 with leucine or valine also produced nonfunctional currents. H91 and W93 were also nonfunctional when replaced with cysteine. These residues (in the "HRW cluster") were located at the C-terminal end of the predicted membrane segment. Regions immediately flanking these two clusters have a number of special properties that show that they are important for anion selectivity, as discussed below.

The currents produced by wild-type mBest 2 exhibit a linear $I-V$ relationship (see Fig. $5 A$ ) and do not have any obvious voltage-dependent or time-dependent gating. Cysteine substitution had no effect on the reversal potential and usually did not affect the shape of the $I-V$ curve or the voltage independence of the current. In two mutations, however, the currents became somewhat inwardly (F80C) or outwardly (R92C) rectifying as a result of cysteine substitution (Qu and Hartzell, 2004). Sulfhydryl reagents usually had no effects on the shape of the $I-V$ curve, but $\mathrm{MTSET}^{+}$made V78C somewhat inwardly rectifying and F80C outwardly rectifying.

\section{MTS reagents have unique effects on residues near the SLIP cluster}

By measuring the modification of cysteine-substituted bestrophin channels by anionic and cationic sulfhydryl reagents, one can deduce not only the accessibility of pore amino acids to the extracellular aqueous environment but also the relative anionic/ cationic selectivity at different amino acid positions (Karlin and Akabas, 1998). Figure $1 B$ shows the magnitude of the steady-state effects of MTS sulfhydryl reagents on cysteine-substituted residues in C69-D104. The magnitude of the effect of MTSES ${ }^{-}$was 


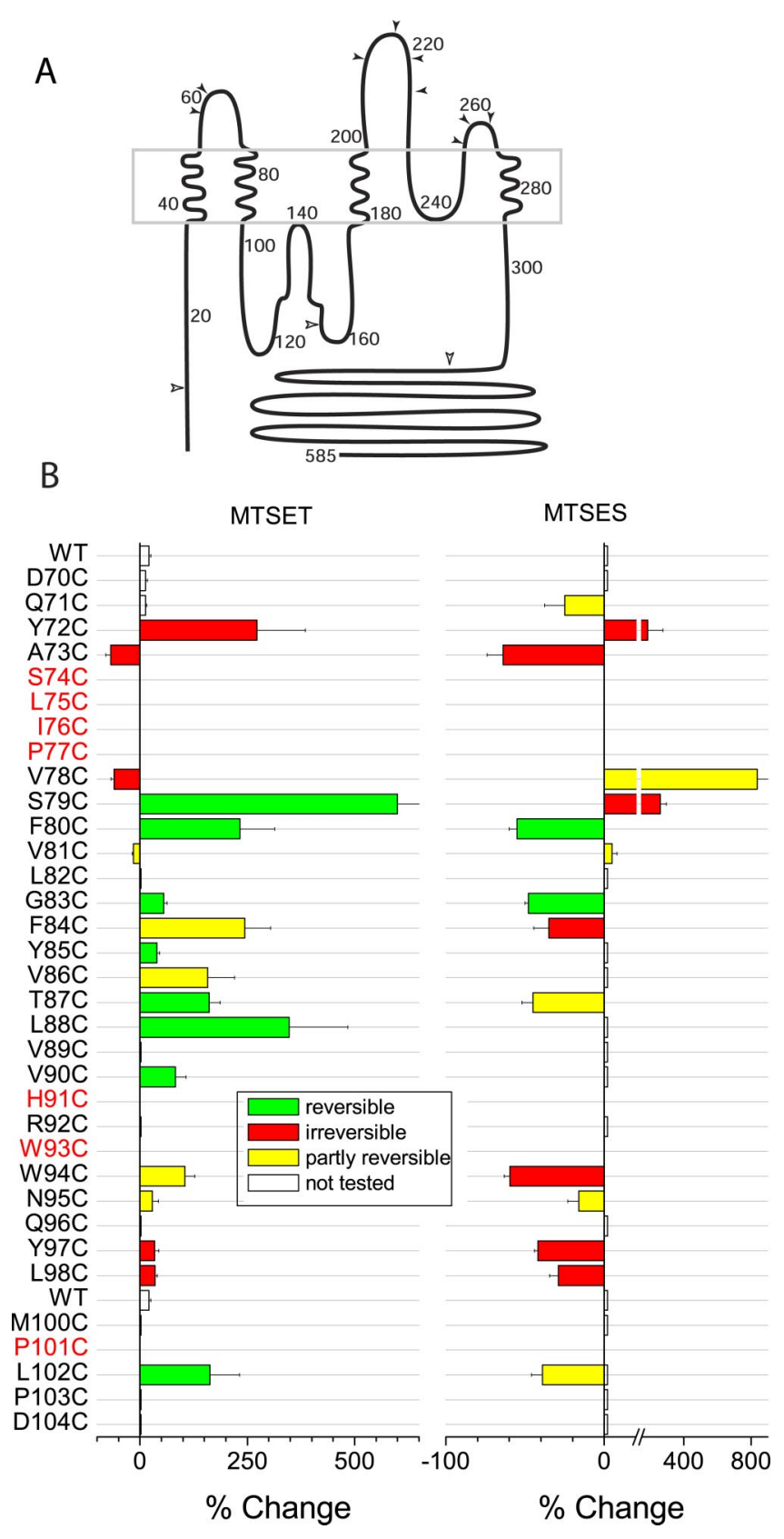

Figure 1. Sulfhydryl modification of mBest2 expressed in HEK293 cells. A, Model of bestrophin topology. A model of the topology of hBest1 proposed by Tsunenari et al. (2003). The topology was determined by insertion of $\mathrm{N}$-glycosylation sites (solid arrows) and TEV protease sites (open arrows). Some TEV protease sites in the C terminus are not shown. $\boldsymbol{B}$, Effects of MTSET $^{+}$(left) and MTSES ${ }^{-}$(right) on cysteine-substituted mBest2 currents. The amino acids indicated on the $y$-axis were replaced one-by-one with cysteine and expressed in HEK293 cells by transient transfection. Currents were recorded by whole-cell patch clamp. Amino acids in red (single letter code, S74-P77, H91, W93) were nonfunctional when replaced with cysteine. The bars indicate the percentage change in amplitude of the mBest2 current at $+100 \mathrm{mV}$ in response to $1 \mathrm{~mm} \mathrm{MTSET}^{+}$or MTSES ${ }^{-}$. The percentage change of the current at $+100 \mathrm{mV}$ was calculated as follows: $\left[100\left(I_{\text {after MTS }}-I_{\text {before MTS }}\right) / I_{\text {before MTS }}\right]$. Positive numbers indicate stimulation, and negative numbers indicate inhibition. MTSET ${ }^{+}$and MTSES ${ }^{-}$were tested on each amino acid (the absence of a bar indicates no effect). The reversibility of the MTS effect was tested by applying 5 mm DTT after washout of the MTS reagent. Red, Not reversible; green, reversible; yellow, partly reversible; white, not tested. Each mutation was tested in 3-12 cells.

very pronounced on residues near the SLIP cluster (Y72, A73, V78, S79, and F80). Because MTSES $^{-}$is anionic and also is intrinsically less reactive than the cationic $\mathrm{MTSET}^{+}$(Stauffer and Karlin, 1994), this result suggests that the residues around the
Table 1. MTS effects at positions flanking the SLIP cluster

\begin{tabular}{cccccccccc}
\hline & Y72 & A73 & S74 & L75 & 176 & P77 & V78 & S79 & F80 \\
\hline MTSET & + & - & & & & & - & + & + \\
MTSES & + & - & & & & & + & + & - \\
\hline
\end{tabular}

SLIP cluster might be involved in anionic selectivity. The effects of the cationic MTSET $^{+}$were distributed over a larger span of residues, but the largest effects were also found around the SLIP cluster.

For most cysteine substitutions that produced currents, MTSET $^{+}$was stimulatory and MTSES $^{-}$was inhibitory (Fig. $1 B$ ). The opposite effects of these oppositely charged reagents suggest that the amplitude of the current is determined by electrostatic interactions between the MTS-modified cysteine in the pore and the permeant anion. Positive charges in the conduction pathway would be expected to attract anions into the channel and increase current, whereas negative charges would tend to repel anions and decrease current. If the effects of $\mathrm{MTSET}^{+}$and $\mathrm{MTSES}^{-}$are electrostatic, one would predict that neutral sulfhydryl reagents would have much smaller effects on the currents. Consistent with this hypothesis, we found that, for the F80C mutation, NEM produced an $11.5 \pm 3.1 \%(n=5)$ decrease in current amplitude at $+100 \mathrm{mV}$ compared with a $233 \pm 81 \%(n=8)$ increase produced by $\mathrm{MTSET}^{+}$.

Notable exceptions to the stimulatory $\mathrm{MTSET}^{+}$-inhibitory MTSES $^{-}$behavior were observed for the residues immediately adjacent to the SLIP cluster (Y72C, A73C, V78C, and S79C). These four amino acids behaved in distinctive ways to sulfhydryl modification by MTSET ${ }^{+}$and MTSES ${ }^{-}$(Fig. $1 B$ ). Not only were the largest magnitude effects of MTS reagents observed with cysteine substituted at these positions, but the MTS effects at positions flanking the SLIP cluster were qualitatively different than their effects on other residues. Furthermore, the effects exhibited an intriguing spatial progression. Y72 and S79 were stimulated by both MTSET $^{+}$and MTSES $^{-}$, Y73 was inhibited by both MTSET $^{+}$and MTSES ${ }^{-}$, and V78 was inhibited by MTSET ${ }^{+}$and stimulated by MTSES $^{-}$, as summarized in Table 1.

The stimulatory effects at positions 72 and 79 and the inhibitory effects at position 73 are likely to be caused by steric or conformational changes rather than electrostatic effects, because the direction and magnitude of the MTSET ${ }^{+}$and MTSES ${ }^{-}$effects are the same at any one of these positions. If the effects were via electrostatic interactions, one would expect the oppositely charged reagents to produce opposite effects. If MTS reagents produce their effects at these positions through steric effects, we predicted that neutral NEM might have a similar effect to the charged sulfhydryl reagents. This was indeed the case for S79C in which NEM stimulated the current $236 \pm 31 \%(n=7)$ compared with a $500 \pm 163 \%(n=8)$ stimulation produced by MTSET ${ }^{+}$.

We also tested the effects of MTSET ${ }^{+}$and MTSES $^{-}$on cells expressing each of the six constructs that produced no currents (S74, L75, I76, P77, H91, and W93) to see whether the MTS reagents could rescue the current. In no case were the MTS reagents able to induce currents.

\section{Irreversibility of MTS effects}

In principle, the effects of MTS reagents should be reversed by reducing agents such as DTT. In Figure $1 B$, the ability of DTT (5 $\mathrm{mM}$ ) to reverse the effects of MTSET ${ }^{+}$and MTSES $^{-}$is indicated by the color of the bars. MTSET ${ }^{+}$was at least partially reversible for most positions, except around the SLIP cluster (Y72C, A73C, 
and V78C) and at Y97C and L98C. In general, MTSES $^{-}$was more difficult to reverse than $\mathrm{MTSET}^{+}$, but the effects of $\mathrm{MTSES}^{-}$on residues around the SLIP cluster (Y72C, A73C, V78C, and S79C) were particularly resistant to reversal. Poor reversibility of MTSES $^{-}$by thiol reagents observed for the R334C mutation in cystic fibrosis transmembrane conductance regulator (CFTR) has been explained in part by electrostatic effects on the reaction between $\beta$-mercaptoethanol and the MTSES $^{-}$-modified cysteine (Smith et al., 2001). This explanation could be applied to S79C because the effect of MTSES ${ }^{-}$is irreversible whereas the effect of $\mathrm{MTSET}^{+}$is reversible. However, the fact that DTT was unable to reverse the effects of either $\mathrm{MTSET}^{+}$or $\mathrm{MTSES}^{-}$at Y72C or $\mathrm{A} 73 \mathrm{C}$ suggests that, for these residues, the situation is more complicated. With R334C CFTR, it was possible to reverse the effect of MTSES ${ }^{-}$by treating with $\beta$-mercaptoethanol at $\mathrm{pH} 9$ to increase the concentration of the reactive thiolate (Smith et al., 2001), but the effects of MTSET ${ }^{+}$or MTSES $^{-}$on Y72C or the effect of MTSES ${ }^{-}$on S79C in mBest2 were not reversed under these same conditions. The fact that the effects of MTS reagents were not readily reversible by DTT at positions $72,73,78$, and 79, which were also among the most reactive residues, indicates that, after sulfhydryl modification, these residues somehow become relatively less accessible to the extracellular solution.

\section{Solvent accessibility of residues near the SLIP cluster}

The rate constants for modification of the cysteine-substituted residues by $\mathrm{MTSES}^{-}$and $\mathrm{MTSET}^{+}$can provide an estimate of the relative solvent accessibility of different residues. Figure 2 shows the second-order rate constants for $\mathrm{MTSET}^{+}$and MTSES $^{-}$modification of residues in C69-D104. The residues that are most rapidly modified by MTSES $^{-}$flank the SLIP cluster (A73C, S79C, and F80C). The rates of modification of these residues by MTSES ${ }^{-}$approach the reaction rates of $\mathrm{MTSES}^{+}$with $\beta$-mercaptoethanol in bulk solution (Stauffer and Karlin, 1994), suggesting that these residues are freely accessible to the extracellular aqueous environment. Residues that react most rapidly to MTSET $^{+}$also flank the SLIP cluster (A73C, S79C, F80C, and $\mathrm{F} 84 \mathrm{C})$. However, the reaction rates of even the most reactive residues with $\mathrm{MTSET}^{+}$are $>10$-fold slower than the rates of $\mathrm{MTSET}^{+}$reaction with $\beta$-mercaptoethanol in bulk solution. This suggests that reaction of the cationic $\mathrm{MTSET}^{+}$may be retarded by the electrostatic environment of this region. From these data, we conclude that A73, S79, F80, and F84 are located in part of the channel that is easily accessible to the extracellular space.

\section{V78C discriminates strongly between anionic and cationic MTS reagents}

By comparing the reaction rates of negatively charged MTSES ${ }^{-}$ and positively charged MTSET $^{+}$with cysteine residues in the pore, it is possible to determine the relative selectivity of each residue for anions and cations (Cheung and Akabas, 1997; Pascual and Karlin, 1998; Wilson et al., 2000). Figure $3 A$ shows the ratio of the second-order rate constants $(\kappa)$ for MTSES $^{-}$and $\mathrm{MTSET}^{+}$modification of nine amino acids in which the magnitude of $\mathrm{MTSET}^{+}$and MTSES ${ }^{-}$effects were large enough to obtain a reliable estimate of their rate constants. For positions 72, 73 , and $79, \kappa_{\mathrm{MTSES}} / \kappa_{\mathrm{MTSET}} \leq 0.5$ (Fig. $3 A$ ). Stauffer and Karlin (1994) showed that MTSET $^{+}$has a much higher intrinsic reactivity with free thiols in bulk solution than does MTSES ${ }^{-}$ $\left(\kappa_{\mathrm{MTSES}} / \kappa_{\mathrm{MTSET}}=0.08\right)$. Thus, cysteine in residues 72,73 , and 79 behave as if they are located in an environment that is either electrostatically neutral or only slightly positively charged. In contrast, $\kappa_{\mathrm{MTSES}} / \kappa_{\mathrm{MTSET}}$ was $>0.5$ for cysteines introduced at

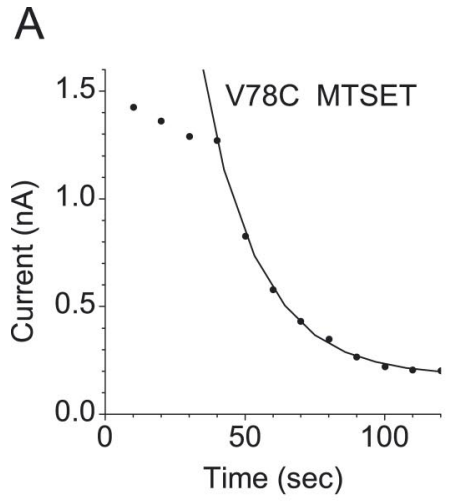

B
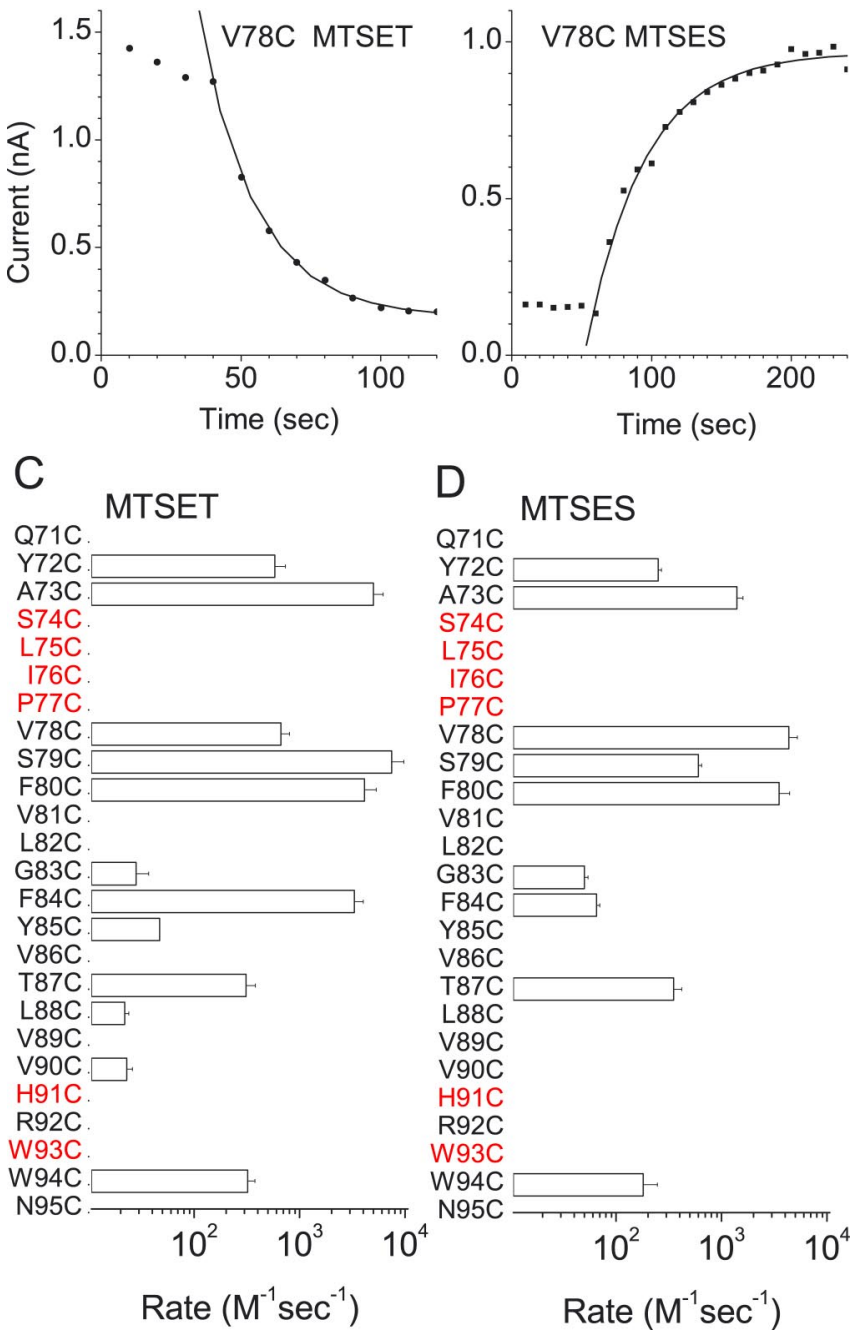

Figure 2. Rate constants of modification of cysteine-substituted amino acids by MTSET ${ }^{+}$ and MTSES ${ }^{-} \cdot \boldsymbol{A}$, Example of rate of change in current amplitude of V78C $\mathrm{mBest} 2$ by MTSET ${ }^{+}$. Currents were elicited by voltage ramps once every $10 \mathrm{~s}$. Filled circles, Current amplitude at $+100 \mathrm{mV}$; solid line, single-exponential fit to the change in current amplitude after exposure to $\mathrm{MTSET}^{+}$. Time constants (seconds) were obtained from the single-exponential fits. $\boldsymbol{B}$, Example of the rate of change in current amplitude of $\mathrm{V} 78 \mathrm{C} \mathrm{mBest} 2$ by MTSES ${ }^{-}$. C, $D$, Rate constants of modification of mBest 2 currents by MTSET ${ }^{+}(\boldsymbol{C})$ and MTSES $^{-}(\boldsymbol{D})$. The amino acids on the $y$-axis were replaced one-by-one with cysteine, and the rate of modification of the current by MTSET ${ }^{+}$ and MTSES ${ }^{-}$was measured as shown in $\boldsymbol{A}$ and $\boldsymbol{B}$. The concentration of MTS reagent was varied between $1 \mathrm{~mm}$ and $10 \mu \mathrm{m}$ to obtain a rate that was reliably measured. Mutations that produced small changes in current were not included in the analysis because of difficulties in accurately quantifying the rate of change. Each mutation was tested in three to nine cells.

positions $78,80,83,87$, and 94 , suggesting that the electrostatic environment around these residues is positively charged (Fig. $3 A$ ). However, of these residues, only V78 and F80 react with MTSES $^{-}$at a rate fast enough for them to be considered readily accessible to the extracellular solution.

The selection of the negatively charged MTSES ${ }^{-}$over the positively charged $\mathrm{MTSET}^{+}$at these residues can be used to infer the relative anion-to-cation selectivity of the pore (Cheung and Akabas, 1997; Pascual and Karlin, 1998; Wilson et al., 2000). The anionic selectivity is greatest for V78. At residues on either side of V78, the relative selectivity declines. From these data, it is possible to estimate the intrinsic anionic selectivity in the pore at each introduced cysteine, assuming that $\mathrm{MTSET}^{+}$and $\mathrm{MTSES}^{-}$are 


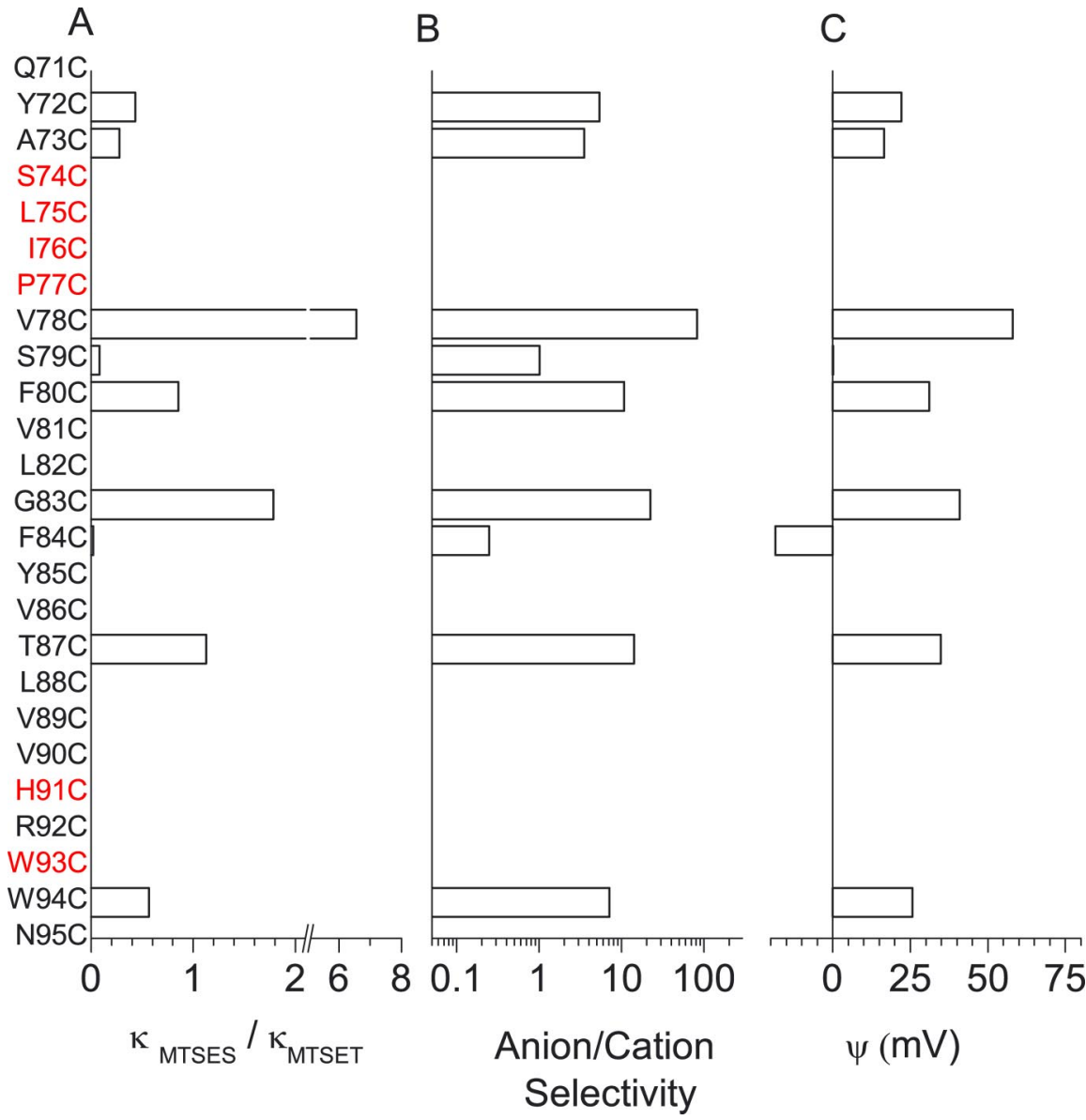

Figure 3. Relative selectivity of different positions for anions over cations. $A$, Relative rate of modification of different cysteinesubstituted residues by MTSES ${ }^{-}$and MTSET $^{+}$was obtained from the rate constants in Figure 2 . $\boldsymbol{B}$, Relative anion/cation selectivity was calculated by normalizing the values in $\boldsymbol{A}$ to the intrinsic rates of modification by MTSES ${ }^{-}$and MTSET ${ }^{+}\left(\kappa^{\prime}{ }_{\text {MTSES }}\right.$ ) $\kappa_{\text {MTSET }}^{\prime}=0.08$ ) in an electrostatically neutral environment of bulk solution (Stauffer and Karlin, 1994). C, Electrostatic potential $(\Psi)$ at each position calculated assuming that selectivity between MTSET ${ }^{+}$and MTSES ${ }^{-}$are caused entirely by electrostatics using the formula in Results. $\Psi$ for $S 79 \mathrm{C}$ is not clearly visible on the plot because it was 0.05

identical with the exception of their charge (Pascual and Karlin, 1998; Wilson et al., 2000). In the absence of any selectivity, one would predict that $\kappa_{\mathrm{MTSES}} / \kappa_{\mathrm{MTSET}}$ would equal the ratio of the reaction rates of MTSES $^{-}$and MTSET $^{+}$with $\beta$-mercaptoethanol in bulk solution $\left(\kappa_{\text {MTSES }}^{\prime} / \kappa_{\text {MTSET }}^{\prime}=0.08\right)$. In the presence of a mechanism that selects between anions and cations, relative anionic/cationic selectivity can be calculated from the ratio $\left(\kappa_{\text {MTSES }} / \kappa_{\text {MTSET }}\right) /\left(\kappa_{\text {MTSES }}^{\prime} / \kappa_{\text {MTSET }}^{\prime}\right)$ (Stauffer and Karlin, 1994). Figure $3 B$ plots the calculated selectivity around each position for which measurements are possible. The position with the highest selectivity is V78.

If selectivity is caused entirely by an electrostatic mechanism, the measured reaction rates should equal $\kappa_{\text {MTSES }}^{\prime} / \kappa_{\text {MTSET }}^{\prime}$ multiplied by a factor that is a function of the intrinsic electrostatic potential, $\Psi:\left(\kappa_{\mathrm{MTSES}} / \kappa_{\mathrm{MTSET}}\right)=\left(\kappa_{\text {MTSES }}^{\prime} / \kappa_{\mathrm{MTSET}}^{\prime}\right)$ $\exp \left[-\left(z_{\mathrm{MTSES}}-z_{\mathrm{MTSET}}\right)(F / R T) \Psi\right]$, where $z_{\mathrm{MTSES}}$ and $z_{\mathrm{MTSET}}$ are the charges of the respective MTS reagents, and $F, R$, and $T$ have their usual thermodynamic meanings. Figure $3 C$ plots the calculated electrostatic potential at the respective residues. At V78, which exhibits the highest anion/cation selectivity, the calculated electrostatic potential is substantial: $65 \mathrm{mV}$.

\section{H91, R92, and W93 are important in channel function}

It is unlikely that MTS reagents report the potential at the modified residue directly because MTS reagents have an estimated length of 6-7 $\AA$ (Wilson et al., 2000). With the additional length of the cysteine side chain, the charged end of the MTS reagent may be reporting the electrostatic potential as far away as $8-9 \AA$. Thus, the positive electrostatic environment in the vicinity of V78 is likely provided by other residues. The only other residues within C69-D104 that might be positively charged are H91 and R92, so we were interested in determining whether these residues contributed to the electrostatics of the pore. Our interest in these residues was heightened by the fact that mutations at positions 91 , 92, and 93 in hBest1 are associated with Best disease.

H91C was nonfunctional in mBest 2 . However, it seems unlikely that H91 contributes significantly to the electrostatics of the pore. The amino acid at position 91 is quite variable among different bestrophins and is not charged in hBest1. Furthermore, the currents induced by the H91T, H91A, and H91K mBest 2 mutants were similar to wild type. Nevertheless, the fact that, in hBest1, the T91I mutation is disease causing reinforces the suspicion that position 91 may be important in other ways.

In contrast to H91, R92 is highly conserved in all bestrophins. To test whether R92 contributes to anion conductance, we changed R92 to histidine, cysteine, or glutamic acid. We predicted that, if R92 plays an electrostatic role in anion conduction, the conductance should be affected by the charge at position 92 in a systematic manner. As expected, the conductance decreased with increasing negativity of the side chain (Fig. $4 A$ ). Although this result is consistent with our prediction, the effects of these mutations could also be attributable to changes in channel gating or expression levels.

If R92 contributes to the electropositivity sensed at V78, we would expect that mutations that reduce the positive charge at position 92 would reduce the rate of modification of V78C by MTSES. R92 was replaced with histidine, which would be less likely to be positively charged than arginine because of its more acidic pKa. As predicted, replacement of R92 with histidine reduced the rate of modification of V78C by MTSES $^{-}$more than fivefold. The rate of MTSES ${ }^{-}$modification for V78C was $4330 \pm$ $890 \mathrm{M}^{-1} \mathrm{~s}^{-1}(n=13)$, whereas the rate for $\mathrm{V} 78 \mathrm{C} / \mathrm{R} 92 \mathrm{H}$ was $825 \pm 23 \mathrm{M}^{-1} \mathrm{~s}^{-1}(n=7)$. However, the interpretation of this experiment is compromised because we do not actually know the charge on the histidine residue in the local environment of the protein. However, evidence that the replacement of arginine with histidine may have altered the charge in the pore was provided by the finding that currents induced by R92 H mBest 2 had different sensitivity to $\mathrm{pH}$ than those of wild-type mBest2 (data not shown). These data, and the fact that R92C, R92S, and R92H are disease-causing mutations in hBest1, support the suggestion that $\mathrm{R} 92$ is an important residue in the function of the mBest 2 channel and may contribute to the electrostatics of the pore.

W93C in hBest1 is also a Best disease-causing mutation. The 

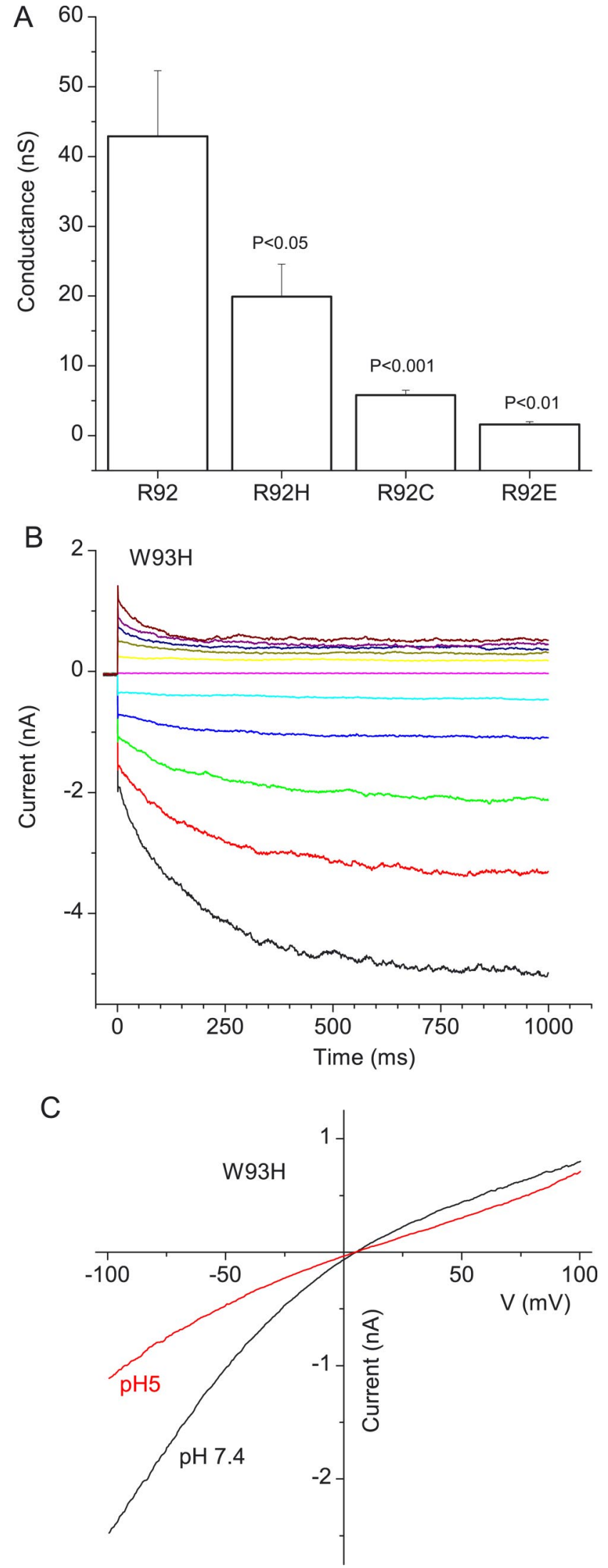

Figure 4. Role of R92 and W93 in selectivity. A, Whole-cell conductances of HEK293 cells transfected with mBest2 constructs in which $\mathrm{R} 92$ was replaced with $\mathrm{H}, \mathrm{C}$, or E. $\boldsymbol{B}$, Timedependent kinetics of currents of $\mathrm{W} 93 \mathrm{H}$ mutant. Superimposed current traces elicited by voltage pulses from a holding potential of $0 \mathrm{mV}$ to voltages between $-100 \mathrm{and}+100 \mathrm{mV}$ in $20 \mathrm{mV}$ steps. C, Current-voltage relationships of W93H current at $\mathrm{pH} 7.4$ and $\mathrm{pH} 5$.
W93C mutation in both mBest 2 and hBest1 is nonfunctional when expressed in HEK cells and is dominant negative (Sun et al., 2002; Qu and Hartzell, 2003). However, replacement of W93 with some other amino acids in mBest 2 produces functional channels. Notably, the W93H mutation exhibited dramatic voltage-dependent gating behavior (Fig. 4B). The current strongly inwardly rectified and had time-dependent activation at negative potentials and time-dependent deactivation at positive potentials. The inward rectification of $\mathrm{W} 93 \mathrm{H}$ was dependent on external $\mathrm{pH}$ within a range that would be expected to change the protonation and charge of histidine (Fig. 4C). This voltagedependent behavior of W93C is consistent with W93 playing a role in channel gating and raises the possibility that the nearby amino acids including H91 and R92 are also involved in channel gating. Overall, the interesting effects of mutations in residues H91, R92, and W93 in mBest2, as well as the occurrence of human disease-causing mutations in corresponding residues in hBest1, point to important roles of these residues in bestrophin channel function.

\section{Possible contributions of R51, R141, and K289 to the pore}

Although the rate of MTSES $^{-}$modification was reduced in the V78C-R92H mutation, the rate was still relatively high, suggesting that basic amino acids in other parts of the protein may provide additional positive charge. We searched for positively charged residues in and near other transmembrane domains that could contribute to the electrostatic potential sensed at V78. Cysteine substitution of R34, R120, R122, R126, K149, R150, K180, R196, R197, or R200 produced essentially wild-type currents, suggesting that these residues are not involved in anion permeation. R125C and R130(C,K,Q, or E) were nonfunctional. These results raised the possibility that $\mathrm{R} 125$ and $\mathrm{R} 130$ could be important, but we did not analyze them further.

Three mutations did produce altered currents: R51C, R141C, and K289C. R51 and K289 are very highly conserved among all bestrophins from Caenorhabditis elegans to humans. R51 is predicted to be at the extracellular end of the first transmembrane domain, and K289 is predicted to be at the cytoplasmic end of the last transmembrane domain. R141 is predicted to be in a cytoplasmic loop and is well conserved, but to a slightly lesser degree.

Cysteine substitution at R51 altered both relative permeability and conductance (Figs. 5B, 6). In wild-type mBest2, the relative permeability of SCN (thiocyanate) to $\mathrm{Cl}\left(P_{\mathrm{SCN}} / P_{\mathrm{Cl}}\right)$ is high and relative conductance $\left(G_{\mathrm{SCN}} / G_{\mathrm{Cl}}\right)$ is low (Figs. $\left.5 A, 6\right)$. With the R51C mutation, $G_{\mathrm{SCN}} / G_{\mathrm{Cl}}$ was 23 -fold greater than wild type $(3.5 \pm 0.25 ; n=7)$, whereas $P_{\mathrm{SCN}} / P_{\mathrm{Cl}}$ was approximately half that of wild type $(4.03 \pm 0.65 ; n=7)$ (Figs. $5 B, 6)$. The R51C mutation was sensitive to $\mathrm{MTSET}^{+}$, as one would expect for an amino acid located close to the extracellular side of the membrane (Fig. 5E). MTSET $^{+}$strongly inhibited the current $(72 \pm 8.9 \%$ inhibition; $n=5$ ) (Fig. 5C), whereas MTSES ${ }^{-}$and NEM had no significant effects. If electrostatic charge at position 51 is important for anion selectivity, one might expect that adding positive charge at R51C by $\mathrm{MTSET}^{+}$modification would increase $\mathrm{Cl}$ conductance. However, the finding that MTSET $^{+}$inhibits the current suggests that MTSET $^{+}$modification may block anion conduction by steric or conformational effects.

Cysteine substitution at K289 also altered relative conductance and permeability (Fig. $5 C$ ). Immediately after changing the extracellular anion from $\mathrm{Cl}^{-}$to $\mathrm{SCN}^{-}, G_{\mathrm{SCN}} / G_{\mathrm{Cl}}$ was greater than wild type $(0.94 \pm 0.17 ; n=5)$, whereas $P_{\mathrm{SCN}} / P_{\mathrm{Cl}}$ was much less than wild type $(3.0 \pm 0.26 ; n=5)$ (Figs. $5 C, 6)$. With time after changing to $\mathrm{SCN}^{-}$, the conductance slowly decreased to a 

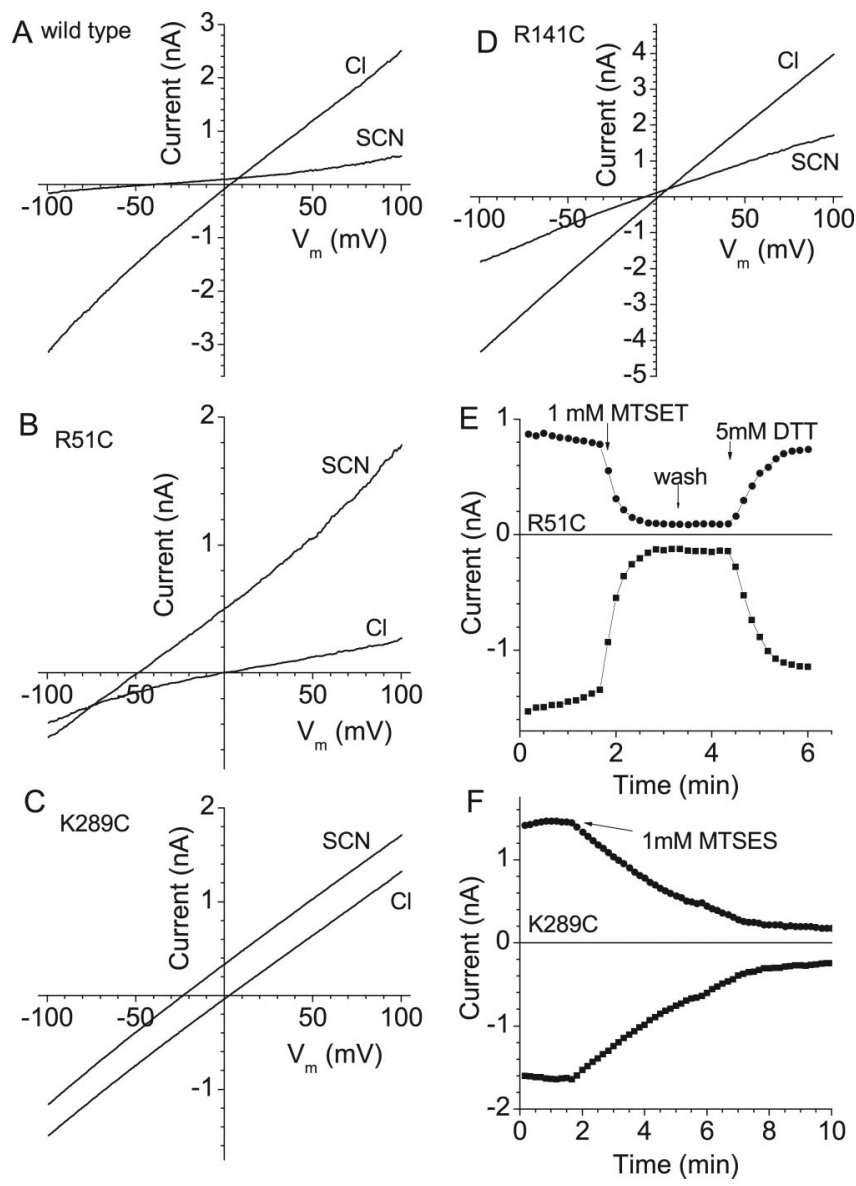

Figure 5. Basic amino acids in other transmembrane domains may contribute to anion selectivity. $\boldsymbol{A}-\boldsymbol{D}$, Current-voltage relationships to voltage ramps for mBest2 wild type $(\boldsymbol{A}), \mathrm{R} 51 \mathrm{C}$ $(\boldsymbol{B}), \mathrm{K} 289 \mathrm{C}(\boldsymbol{C})$, and R141C (D). E, $\boldsymbol{F}$, Time course of effects of $1 \mathrm{~mm}$ MTS reagents on R51C (E) and $\mathrm{K} 289 \mathrm{C}(\boldsymbol{F})$.

level near wild type with a time constant of $\sim 45 \mathrm{~s}$, presumably as a consequence of accumulation of $\mathrm{SCN}^{-}$in the cell. K289C responded very slowly to MTS reagents (Fig. $5 F$ ), as would be expected for an amino acid located at the cytoplasmic side of the pore. Although both MTSET $^{+}$and MTSES $^{-}$had large effects on the K289C current MTSET $^{+}$inhibited the current $84 \pm 4 \%$, and MTSES $^{-}$inhibited the current $83 \pm 3 \%$ ), the rate constants for $\mathrm{MTSET}^{+}$and MTSES $^{-}$modification were both very slow, $<10 \pm 2 \mathrm{M}^{-1} \mathrm{~s}^{-1}$. This is consistent with $\mathrm{K} 289$ being located in a portion of the channel facing the cytoplasm. To test this idea further, we asked whether reducing the charge at K289 would alter the positivity sensed at V78, as we did for R92. Of the K289 substitutions that were made, only the $\mathrm{K} 289 \mathrm{H}$ mutation was functional, but the V78C-K289H double mutation was nonfunctional. Thus, it was not possible to determine whether K289 contributed to the positivity detected at V78.

The R141C mutation also had altered relative conductance and permeability: $G_{\mathrm{SCN}} / G_{\mathrm{Cl}}$ was less than wild type $(0.35 \pm 0.06$; $n=6)$, whereas $P_{\mathrm{SCN}} / P_{\mathrm{Cl}}$ was much less than wild type $(1.5 \pm$ $0.16 ; n=6$ ) (Figs. 5D, 6). The R141C mutation was insensitive to extracellular sulfhydryl reagents, suggesting that it was on the cytoplasmic side of the pore.

\section{Cysteine substitutions alter relative anion conductance and permeability}

Figure 6 summarizes the effects of cysteine substitutions on the relative anion permeability and conductance of the channel. Cys- teine substitution of many of the residues in the predicted transmembrane domain increased $G_{\mathrm{SCN}} / G_{\mathrm{Cl}}$ and decreased $P_{\mathrm{SCN}} / P_{\mathrm{Cl}}$. In Figure 6, wild-type mBest 2 is represented by the bars at C69 and C99, which are normally cysteine. In wild-type mBest2, the relative permeability of SCN to $\mathrm{Cl}\left(P_{\mathrm{SCN}} / P_{\mathrm{Cl}}\right)$ is high (9.4), and the relative conductance $\left(G_{\mathrm{SCN}} / G_{\mathrm{Cl}}\right)$ is low $(0.15)$. For 10 residues, cysteine substitution decreased $P_{\mathrm{SCN}} / P_{\mathrm{Cl}}$ and increased $G_{\mathrm{SCN}} / G_{\mathrm{Cl}}$ (Fig. $6 C$, green bars). These residues were located within the predicted transmembrane domain, between V78 and R92, between the SLIP and HRW clusters. Cysteine substitution of residues $\mathrm{C}$ terminal to W94 had only a sporadic effect.

To illustrate the overall effect of cysteine substitution on the biophysical properties of the channel, we plotted the ratio of relative permeability to relative conductance for each position and normalized the ratio to wild type of 1.0 (Fig. $6 \mathrm{C}$ ). The ratio of relative permeability to relative conductance is considered to be a measure of the relative affinity of the channel for the permeant ion (Läuger, 1973; Halm and Frizzell, 1992). Green bars indicate values that were significantly different from wild type. Residues that differed significantly from wild type in both relative conductance and relative permeability were located between V78 and R92. This supports the notion that the pore-forming domain of the channel is located in this region and that the SLIP and HRW clusters are important in channel function. V78C, S79C, F80C, and $\mathrm{F} 84 \mathrm{C}$, which were among the residues most readily modified by $\mathrm{MTSET}^{+}$, also emerged as residues whose relative permeability and conductance were changed significantly by cysteine substitution, thus establishing the importance of these residues in ion channel function.

\section{Discussion}

\section{A model of mBest2 TMD2}

Our data provide additional support for the participation of TMD2 in forming the pore of mBest2. Among 17 vertebrate bestrophins found in GenBank, all have sequences identical to mBest2 from amino acid I76 to W94 with the exception of V78, which can also be I, L, or M, and S79, which can also be T. There is somewhat more divergence when one examines all eukaryotic bestrophins, but still the conservation is very high. All 60 eukaryotic bestrophins fit the pattern: [IVL]-P-[IVL]-[polar]-F[MVL]-L-G-F-[YF]-V-[STAN]-X-[IV]-[hydrophobic]-X-R-W, where $X$ indicates any amino acid. It should be emphasized that the $\mathrm{Cl}^{-}$channel properties of most of the bestrophins have not been studied directly and that sequence variability might reflect important functional differences. Nevertheless, the identity of residues P77, F80, L82, G83, F84, Y85, V86, R92, and W93 across phyla suggests that these residues are critical for bestrophin function.

The observation that mutations within TMD2 alter the relative conductance and permeability of the channel to anions is supportive of the hypothesis that TMD2 participates in the formation of the pore of the channel. The residues that react most rapidly with anionic sulfhydryl reagents are A73C, V78C, S79C, and $\mathrm{F} 80 \mathrm{C}$, suggesting that these residues may form an outer vestibule. Of the residues we studied, V78C exhibits the highest anionic selectivity as measured by rates of modification by anionic and cationic MTS reagents, suggesting that V78 may participate in anionic selectivity as well. T87C and W94C also exhibited significant anionic selectivity, but their rates of modification were considerably slower than that of V78C, consistent with them being located deeper in the pore.

The pattern of MTS modification does not appear compatible with an $\alpha$ helical structure because clusters of adjacent residues 
are often affected similarly. For example, residues $78-80$ react with similar rates to MTS reagents. Such a pattern is compatible with this region having an extended structure. Beyond F80, if one looks only at the modification by MTSES $^{-}$, there may be a hint of a helical structure because only every third residue is modified. However, this pattern is not reflected by MTSET $^{+}$ modification.

In both CFTR (Smith et al., 2001) and ligand-gated anion channels (Keramidas et al., 2002), positively charged amino acids have been shown to be involved in anion permeation through the pore. However, the only amino acids in TMD2 of mBest 2 that are likely to be positively charged are H91 and R92. Our data show that the highly conserved R92 is likely to be a critical residue because mBest 2 macroscopic conductance is related to the charge at this position. Furthermore, the effects of membrane-impermeant MTS reagents on R92C are negligible, suggesting that this residue is not easily accessible to the extracellular space and may be located at or beyond a constriction in the pore. However, because W94C is modified at moderate rates by MTS reagents implies that the inaccessibility of R92 may also be explained by the side chain being in an inaccessible local environment.

It seems unlikely that the second transmembrane domain forms the pore by itself, because mutation of residues in other parts of the protein also affects channel function. In particular, mutation of several positively charged residues, namely R51, $\mathrm{R} 141$, and K289, alter relative permeability and conductance of the channel.

\section{Anionic selectivity of bestrophins}

The experiments reported here add additional support to the proposal that bestrophins are $\mathrm{Cl}$ channels. Several different lines of evidence now make the idea that bestrophins can function as $\mathrm{Cl}$ channels inescapable: bestrophins expressed in different cell lines produce the same kind of $\mathrm{Ca}$-activated $\mathrm{Cl}$ currents (Sun et al., 2002; Qu and Hartzell, 2003); different bestrophin paralogs produce currents with different characteristics (Sun et al., 2002; Tsunenari et al., 2003); expression of dominantnegative mutants of bestrophin inhibit currents induced by expression of the wild-type bestrophin; and point mutations in both hBest 1 and mBest 2 produce changes in $\mathrm{Cl}$ channel function (Tsunenari et al., 2003; Qu and Hartzell, 2004; Qu et al., 2004). Because it is generally agreed that the selectivity of a channel is determined by the channel pore, the ability to change the selectivity by a mutation provides strong evidence that bestrophin is responsible for forming the channel (Pusch, 2004).

Partitioning of an ion between bulk water and the channel pore depends on a balance between electrostatic interactions of the permeant ion with the channel pore and the free energy of hydration of the ion. The partitioning of the ion into the pore is tested in 5-14 cells.
B

C

C
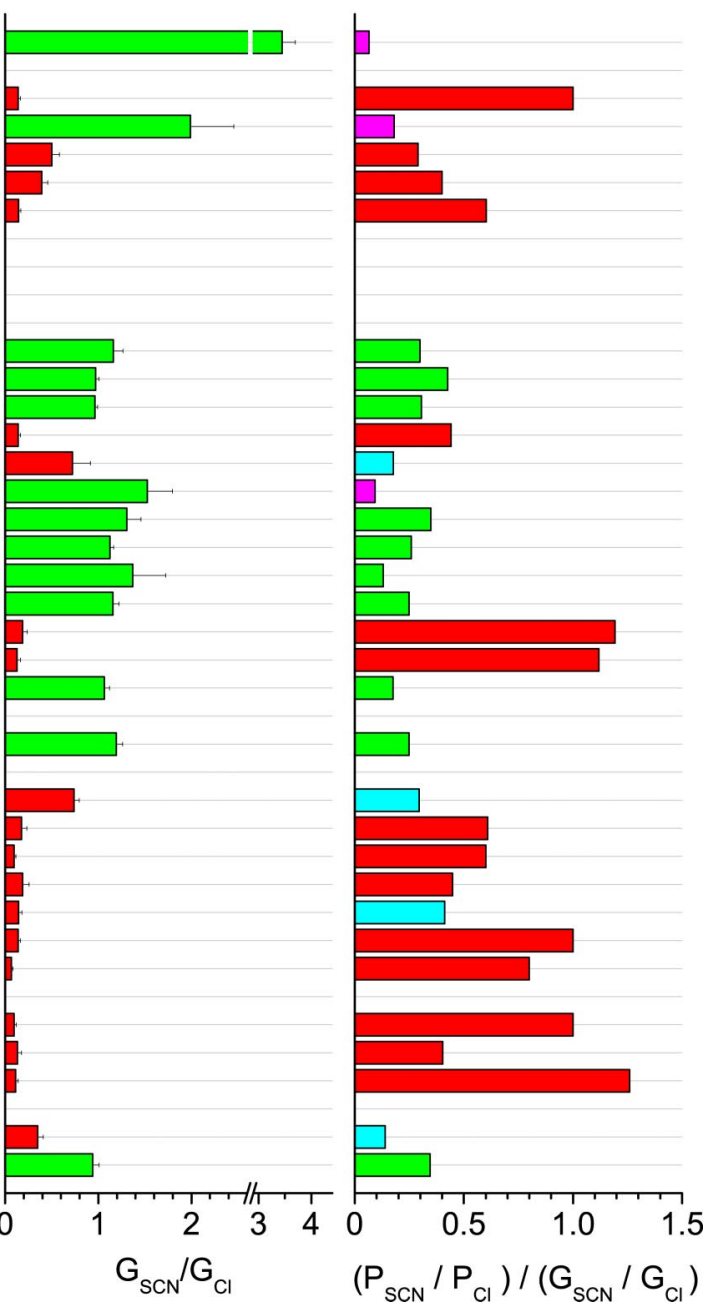

Figure 6. Effects of replacement of amino acids in $\mathrm{mBest2}$ with cysteine on $\mathrm{SCN}^{-}$permeability and conductance relative to

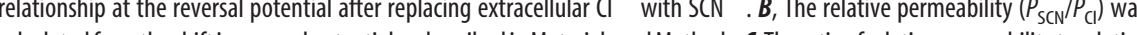
calculated from the shift in reversal potential as described in Materials and Methods. $C$, The ratio of relative permeability to relative (a) magenta bars represent mutations that significantly affected only permeability or conductance, respectively. Each mutation was

dominated by hydration energy if the electrostatic interaction with the channel is weak. In this case, the permeability of larger ions, which are more easily dehydrated, is greater than that of smaller ions and the channel exhibits a lyotropic anion permeability sequence with $\mathrm{SCN}^{-}>\mathrm{NO}_{3}{ }^{-}>\mathrm{I}^{-}>\mathrm{Br}^{-}>\mathrm{Cl}^{-}>\mathrm{F}^{-}$ (Eisenman and Horn, 1983). In contrast, if electrostatic interactions dominate, smaller anions $\left(\mathrm{F}^{-}, \mathrm{Cl}^{-}\right)$are generally more permeant than larger ones $\left(\mathrm{SCN}^{-}, \mathrm{NO}_{3}{ }^{-}, \mathrm{I}^{-}\right)$. Because the permeability sequence for $\mathrm{mBest} 2$ follows the lyotropic sequence (Qu et al., 2004), hydrophobic interactions must be important. This may explain why the pore of the channel is dominated by hydrophobic amino acids. Anion permeation in bestrophins can be explained by anion partition into a hydrophilic channel that has a dielectric constant slightly less than water $(\varepsilon=\sim 20)$. It seems to be a common property of $\mathrm{Cl}^{-}$channels, in contrast to cation channels, that the determinants of permeation are distributed over a larger stretch of amino acids. This may be because the structural determinants of pore formation in a channel that is 
relatively nonselective are not as rigid as the requirements for $\mathrm{K}^{+}$ ion coordination in a pore that is highly selective for $\mathrm{K}^{+}$over other cations.

\section{Mechanisms of macular degeneration}

More than 85 mutations in hBest1 have now been linked to Best vitelliform macular dystrophy. Seventeen of these mutations are found between amino acids 73 and 104. Each of the key amino acids that we identified as playing a potential role in anion permeation, namely F80, F85, R92, and W93, have diseaseassociated mutations and are identical in all eukaryotic bestrophins. Thus, our data provide important insights into the mechanisms of Best macular dystrophy. Dysfunction of the bestrophin $\mathrm{Cl}$ channel certainly is likely to be an initial defect in the disease. Defects in $\mathrm{Cl}$ channel function, whether playing a role in regulation of cell membrane potential or cell volume at the plasma membrane or regulating vesicular $\mathrm{pH}$ and trafficking in intracellular membranes, would certainly have serious effects on cellular function.

\section{References}

Bakall B, Marmorstein LY, Hoppe G, Peachey NS, Wadelius C, Marmorstein $\mathrm{AD}$ (2003) Expression and localization of bestrophin during normal mouse development. Invest Ophthalmol Vis Sci 44:3622-3628.

Cheung M, Akabas MH (1997) Locating the anion-selectivity filter of the cystic fibrosis transmembrane conductance regulator (CFTR) chloride channel. J Gen Physiol 109:289-299.

Clapham DE (1998) The list of potential volume-sensitive chloride currents continues to swell (and shrink). J Gen Physiol 111:623-624.

Eisenman G, Horn R (1983) Ionic selectivity revisited: the role of kinetic and equilibrium processes in ion permeation through channels. J Membr Biol 76:197-225.

Fischmeister R, Hartzell C (2004) Volume-sensitivity of the bestrophin family of chloride channels. J Physiol (Lond) 552 2:477-491.

Gallemore RP, Hughes BA, Miller SS (1997) Retinal pigment epithelial transport mechanisms and their contributions to the electroretinogram. Prog Retin Eye Res 16:509-566.

Halm DR, Frizzell RA (1992) Anion permeation in an apical membrane chloride channel of a secretory epithelial cell. J Gen Physiol 99:339-366.

Hartzell HC, Qu Z, Putzier I, Artinian L, Chien L-T, Cui Y (2005) Looking chloride channels straight in the eye: bestrophins, lipofuscinosis, and retinal degeneration. Physiology 20:292-302.

Jentsch TJ, Stein V, Weinreich F, Zdebik AA (2002) Molecular structure and physiological function of chloride channels. Physiol Rev 82:503-568.

Karlin A, Akabas MH (1998) Substituted-cysteine accessibility method. Methods Enyzmol 293:123-145.

Keramidas A, Moorhouse AJ, Pierce KD, Schofield PR, Barry PH (2002) Cation-selective mutations in the M2 domain of the inhibitory glycine receptor channel reveal determinants of ion-charge selectivity. J Gen Physiol 119:393-410.

Kuruma A, Hartzell HC (2000) Bimodal control of a $\mathrm{Ca}^{2+}$-activated $\mathrm{Cl}^{-}$ channel by different $\mathrm{Ca}^{2+}$ signals. J Gen Physiol 115:59-80.

Läuger P (1973) Ion transport through pores: a rate-theory analysis. Biochim Biophys Acta 311:423-441.

Marmorstein AD, Mormorstein LY, Rayborn M, Wang X, Hollyfield JG, Petrukhin K (2000) Bestrophin, the product of the Best vitelliform macular dystrophy gene (VMD2), localizes to the basolateral membrane of the retinal pigment epithelium. Proc Natl Acad Sci USA 97:12758-12763.
Marquardt A, Stohr H, Passmore LA, Kramer F, Rivera A, Weber BH (1998) Mutations in a novel gene, VMD2, encoding a protein of unknown properties cause juvenile-onset vitelliform macular dystrophy (Best's disease). Hum Mol Gen 7:1517-1525.

Neher E (1992) Ion channels for communication between and within cells. Science 256:498-502.

Pascual JM, Karlin A (1998) Delimiting the binding site for quaternary ammonium lidocaine derivatives in the acetylcholine receptor channel. J Gen Physiol 112:611-621.

Penfold PL, Madigan MC, Gillies MC, Provis JM (2001) Immunological and aetiological aspects of macular degeneration. Prog Retin Eye Res 20:385-414.

Petrukhin K, Koisti MJ, Bakall B, Li W, Xie G, Marknell T, Sandgren O, Forsman K, Holmgren G, Andreasson S, Vujic M, Bergen AAB, McGartyDugan V, Figueroa D, Austin CP, Metzker ML, Caskey CT, Wadelius C (1998) Identification of the gene responsible for Best macular dystrophy. Nat Genet 19:241-247.

Pusch M (2004) $\mathrm{Ca}^{2+}$-activated chloride channels go molecular. J Gen Physiol 123:323-325.

Qu Z, Hartzell HC (2000) Anion permeation in $\mathrm{Ca}^{2+}$-activated $\mathrm{Cl}^{-}$channels. J Gen Physiol 116:825-844.

Qu Z, Hartzell HC (2003) Two bestrophins cloned from Xenopus laevis Oocytes express Ca-activated Cl currents. J Biol Chem 278:49563-49572.

Qu Z, Hartzell HC (2004) Determinants of anion permeation in the second transmembrane domain of the mouse bestrophin-2 chloride channel. J Gen Physiol 124:371-382.

Qu Z, Fischmeister R, Hartzell HC (2004) Mouse bestrophin-2 is a bona fide $\mathrm{Cl}^{-}$channel: identification of a residue important in anion binding and conduction. J Gen Physiol 123:327-340.

Smith SS, Liu X, Zhang ZR, Sun F, Kriewall TE, McCarty NA, Dawson DC (2001) CFTR: covalent and noncovalent modification suggests a role for fixed charges in anion conduction. J Gen Physiol 118:407-432.

Stauffer DA, Karlin A (1994) Electrostatic potential of the acetylcholine binding sites in the nicotinic receptor probed by reactions of binding-site cysteines with charged methanethiosulfonates. Biochemistry 33:6840-6849.

Strauss O (2005) The retinal pigment epithelium in visual function. Physiol Rev 85:845-881.

Sun H, Tsunenari T, Yau K-W, Nathans J (2002) The vitelliform macular dystrophy protein defines a new family of chloride channels. Proc Natl Acad Sci USA 99:4008-4013.

Tsunenari T, Sun H, Williams J, Cahill H, Smallwood P, Yau K-W, Nathans J (2003) Structure-function analysis of the bestrophin family of anion channels. J Biol Chem 278:41114-41125.

Wajima R, Chater SB, Katsumi O, Mehta MC, Hirose T (1993) Correlating visual acuity and electrooculogram recordings in Best's disease. Ophthalmologie 207:174-181.

Weber BHF, Krämer F (2002) VMD2 Mutation Database. http://www. uni-wuerzburg.de/humangenetics/vmd2.html. Accessed April 27, 2006.

White K, Marquardt A, Weber BHF (2000) VMD2 mutations in vitelliform macular dystrophy (Best disease) and other maculopathies. Hum Mutat 15:301-308

Wilson GG, Pascual JM, Brooijmans N, Murray D, Karlin A (2000) The intrinsic electrostatic potential and the intermediate ring of charge in the acetylcholine receptor channel. J Gen Physiol 115:93-106.

Yardley J, Leroy BP, Hart-Holden N, Lafaut BA, Loeys B, Messiaen LM, Perveen R, Reddy MA, Bhattacharya SS, Traboulsi E, Baralle D, De Laey JJ, Puech B, Kestelyn P, Moore AT, Manson FD, Black GC (2004) Mutations of VMD2 splicing regulators cause nanophthalmos and autosomal dominant vitreoretinochoroidopathy (ADVIRC). Invest Ophthalmol Vis Sci 45:3683-3689. 